\title{
Europe's space funds feel the squeeze...
}

[MUNICH] Space scientists in Europe are trying to convince member states of the European Space Agency (ESA), whose space ministers are due to meet next month, to lift a threeyear funding 'cap' on the agency's science programme.

The cap has not only significantly eroded the programme, but also helped to plunge it into its most serious financial crisis. According to Roger Bonnet, ESA's space science director, if the cap is not lifted, some programmes in ESA's long-term Horizons 2000 science plan may have to be abandoned.

At present, ESA's member states are divided roughly equally in their willingness to lift the cap. But since a unanimous decision is required, the cap is likely to remain.

Horizons 2000 includes four approved large 'cornerstone' missions, each costing about ECU650 million (US\$786 million), balanced by medium-sized missions at about half this cost in various disciplines.

The programme was approved by ESA in 1994 when a continuous growth in budget was foreseen. Two years later, however, the space ministers, facing economic difficulties at home, decided to hold the budget constant for three years. There was to be no increase for inflation, and this would automatically be extended for a further two years unless ministers agreed unanimously to raise it again.

ESA has since introduced extensive costcutting and efficiency measures. It has also been dogged by bad luck. In particular, two important Solar System missions — ESA's Cluster and Russia's Mars 96, with many ESA instruments on board - were lost on launch in 1996 (see Nature 381, 541; 1996).

To maintain a Horizons programme serving all scientific disciplines, Bonnet decided, with the approval of the ESA council, to relaunch Cluster with existing spares, and to

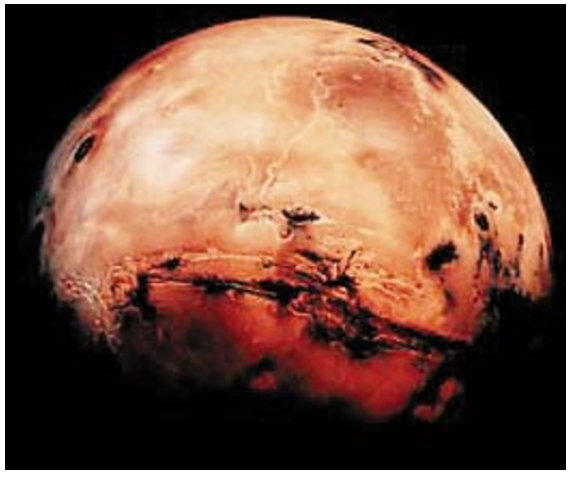

To Mars? The unforeseen Mars Express weighs heavily on ESA's already stretched purse-strings.

develop the Mars Express concept.

The second of these would carry duplicates of the Mars 96 instruments, and complement the flotilla of spacecraft that the US space agency NASA is sending to Mars in the next few years. The cost of Mars Express has been capped at ECU150 million.

But such unplanned-for expenses have placed further strain on ESA's budget. And more trouble could be ahead. The launch of the X-ray astronomy cornerstone mission XMM, scheduled for 1999, has been delayed for six months by technical problems.

The delay will add to the costs because of the need to keep teams together, while uncertainty hangs over Russia's ability to make good on its promise to provide a free Proton launch for the gamma-ray astronomy mission Integral, scheduled for launch in 2001.

An alternative Ariane-5 launch remains possible, but would cost ECU150 million. Also, ESA's new policy of insuring science missions will probably increase some launch costs. Insurance of ECU18 million is likely be taken out for the 2003 Cluster relaunch.

Budget constraints have strained relation-

\section{while UK scientists seek private backing}

[MUNICH] British space

scientists are making an urgent appeal to private funders to support a small equipment package that would land on the surface of Mars as part of the Mars Express mission.

The Mars Express spacecraft was originally developed to carry instruments duplicating those lost when the Russian Mars 96 mission, intended to analyse the martian atmosphere, failed on launch in 1996. At an early stage, however, its scope was broadened to include a

lander to transmit data on the atmosphere and the planet's surface back to Earth.

The UK-led lander, called Beagle, has been approved for inclusion, but only if its principal investigator, Colin Pillinger, director of the Planetary Sciences Research Institute at the Open University in Milton Keynes, can raise private funding

Estimated to cost around $£ 25$ million (US $\$ 43$ million), Beagle would look in particular for evidence of life or former life on the planet.
But its prospects appear bleak. The Particle Physics and Astronomy Research Council has said it cannot afford to contribute, and Pillinger has only until the end of October to present his financial plan to ESA.

The launch of Mars Express is scheduled for 2003 and cannot be delayed, as it depends on planetary alignments. If Pillinger cannot obtain funding, ESA is likely to close the option on the feasibility studies of Mars Express, due to start in November. A.A. ships between Solar System scientists and astrophysicists, who until now have worked relatively happily together within ESA's consensus-driven system of programming.

To make further savings to accommodate Solar System missions, Bonnet has suggested combining two astrophysics missions that are both in the design phase: the infrared cornerstone mission FIRST, and the medium-sized microwave background mission Planck, both approved in 1997.

Bonnet's original plan to place both missions in a single container on the same launch vehicle was rejected in the summer by ESA's science programme committee as restricting the science of each mission (see Nature 387, 639; 1997).

ESA is now assessing the less radical option of keeping the two missions physically separate on the same launch, and delaying launch to 2007 - four years after the scheduled Planck launch and two years after FIRST's scheduled launch - in order to spread costs.

The merger debate caused uproar in the astrophysics community and triggered a wave of discontent. "The scientific community was alarmed by the single-minded effort to save money through complete merging of two missions with different purposes," says Reinhard Genzel, a director of the Max Planck Institute for Extraterrestrial Physics in Garching, and principle investigator for one of the Planck instruments. "It was obvious to everyone what a high risk this would be."

Pleased that a complete merger has been rejected, Genzel still feels that any delay forced on Planck "is unfair", because both Planck and FIRST were selected through ESA's normal mechanism of peer-review competition, while Mars Express "came in the back door".

"ESA procedures are breaking down," he says, pointing out that Mars Express was originally approved on the understanding that it would not affect the rest of the Horizons programme.

Bonnet says he will do his best to keep Mars Express on track, as he is determined to maintain a programme balanced between space science disciplines. But he emphasizes that no fully approved Horizons 2000 mission will be abandoned.

Scientists at ESTEC, ESA's scientific and technical arm, recently passed a vote of no confidence in the science directorate's management, having become frustrated by the lack of decision-making.

Only a budget increase would diffuse the tensions and allow all disciplines to be satisfied. But reestablishing a link with inflation in next month's 1999 budget discussions seems unlikely, as too many member states remain set against it.

Alison Abbot 